\title{
Long-Lasting Complete Remission of Small-Cell Carcinoma of the Pancreas with Carboplatin and Etoposide Complicated by Gallbladder Adenocarcinoma Diagnosed during Follow-Up
}

\author{
Konstantinos Tyriakidis $^{a}$ Sylvia Stadlmann ${ }^{\mathrm{b}}$ Stefanie Pederiva ${ }^{a}$ \\ aDepartment of Medical Oncology, Cantonal Hospital Baden, Baden, Switzerland; ${ }^{b}$ Institute \\ of Pathology, Cantonal Hospital Baden, Baden, Switzerland
}

\section{Keywords}

Carboplatin · Complete remission · Etoposide · Pancreatic cancer · Small-cell carcinoma

\begin{abstract}
Small-cell carcinoma of the pancreas (PSCC) is a highly aggressive neoplasia with a dismal prognosis. It is extremely rare, with only a few cases reported in the literature. There is a paucity of clinical data to guide management and since the disease is mainly diagnosed at an advanced stage standard treatment consists of chemotherapy based upon treatment protocols used for small-cell lung cancer. We report the case of a female diagnosed with PSCC who achieved complete clinical remission after treatment with carboplatin and etoposide. During a 3-year follow-up the patient developed a gallbladder adenocarcinoma that was treated by surgical resection but relapsed within 20 months with widespread hematogenous metastasis.
\end{abstract}

(C) 2021 The Author(s).

Published by S. Karger AG, Basel

\section{Introduction}

Extrapulmonary small-cell carcinoma is a rare neoplasm characterized by an aggressive disease course and poor prognosis. It can arise at multiple organ sites and resembles smallcell lung cancer (SCLC) with regards to pathological features but also clinical behavior. Due to a lack of relevant data, treatment and classification is extrapolated from the management of SCLC. In the case of locoregional disease and depending on the organ site, various multimodality treatment approaches have been suggested. For patients with extensive disease, 
Fig. 1. Complete remission of a PSCC arising in the pancreatic head after treatment with carboplatin and etoposide.

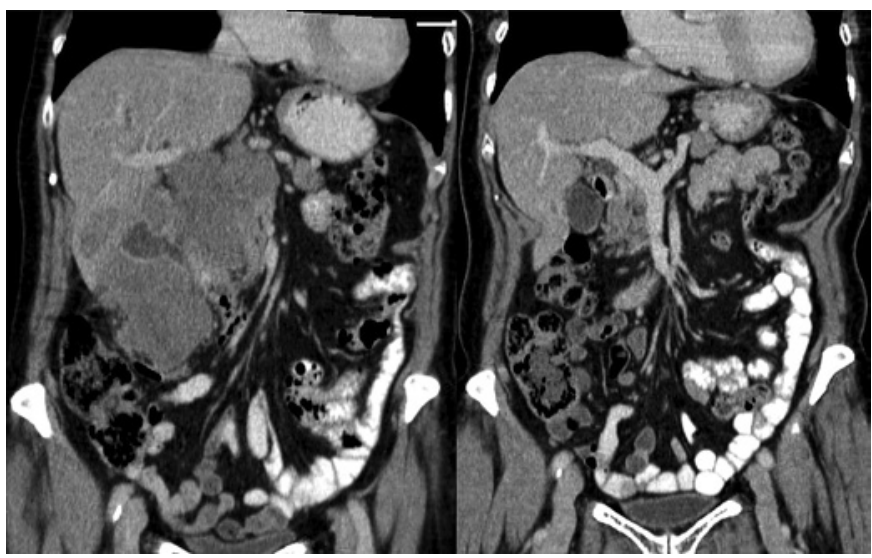

chemotherapy in analogy to treatment of SCLC has been applied in the vast majority of reported cases. Small-cell carcinoma of the pancreas (PSCC) is extremely rare, with only a few cases reported in the literature so far, although it has been postulated that due to its aggressive clinical course some patients may die early and thus remain undiagnosed. Furthermore, patients with PSCC may be falsely classified as SCLC. We report the case of a 74-year-old woman who was diagnosed with PSCC that completely responded to carboplatin and etoposide but developed metastatic adenocarcinoma of the gallbladder during follow-up.

\section{Case Presentation}

A 74-year-old female presented to her family doctor with a 3-week history of progressive abdominal discomfort and pain in her upper abdomen. She also reported fatigue, anorexia, and dizziness. The patient's history consisted of osteoporosis treated with bisphosphonates and hypothyroidism substituted with levothyroxine. Her family history included colorectal cancer (brother) and laryngeal cancer (father). No history of smoking or alcohol consumption was reported.

Ultrasonography revealed a large hypoechogenic mass in the right upper abdomen. A subsequent computed tomography (CT) scan showed a large tumor $(16 \times 11 \mathrm{~cm})$ arising from the pancreatic head and infiltrating the liver without any evidence of thoracic manifestation (Fig. 1). Histopathological examination of a percutaneous ultrasound-guided biopsy revealed small-cell carcinoma (Fig. 2A) with a diffuse expression of synaptophysin (Fig. 2B), CDX2 in almost all tumor cells, and negativity for CK7 and CK20 expression. TTF-1 was weakly expressed in single tumor cells. The Ki-67 proliferation index was 70\%.

The patient was referred to our oncology department for further evaluation and management. Laboratory examination revealed the following elevated enzymes: aspartate transaminase $741 \mathrm{U} / \mathrm{L}$ (normal range 10-35), alanine transaminase $800 \mathrm{U} / \mathrm{L}$ (normal range 10-35), alkaline phosphatase 741 U/L (normal range 35-105), gamma-glutamyl-transferase 1,107 U/L (normal range 6-42), bilirubin $25 \mu \mathrm{mol} / \mathrm{L}$ (normal <21), and lactate dehydrogenase 1,081 U/L (normal range 135-214). The tumor markers carbohydrate antigen 19-9 and carcinoembryonic antigen were within the normal ranges $(28.8 \mathrm{mU} / \mathrm{L}$ and $0.6 \mu \mathrm{g} / \mathrm{L}$, respectively).

Due to extensive disease and after discussion in a multidisciplinary tumor board a palliative systemic chemotherapy was recommended. The patient's performance status (PS) was evaluated according to the Eastern Cooperative Oncology Group (ECOG) and deteriorated rapidly from grade 1 to grade 2 within 1 week of presentation. Chemotherapy with carboplatin and etoposide was initiated 5 weeks after the onset of symptoms. We applied 6 cycles 


\section{Case Reports in Oncology}

\begin{tabular}{l|l}
\hline Case Rep Oncol 2021;14:365-370 \\
\hline DOI: 10.1159/000514048 & $\begin{array}{l}\text { @ 2021 The Author(s). Published by S. Karger AG, Basel } \\
\text { www.karger.com/cro }\end{array}$ \\
\hline
\end{tabular}

Tyriakidis et al.: Complete Remission of Small Cell Carcinoma of the Pancreas
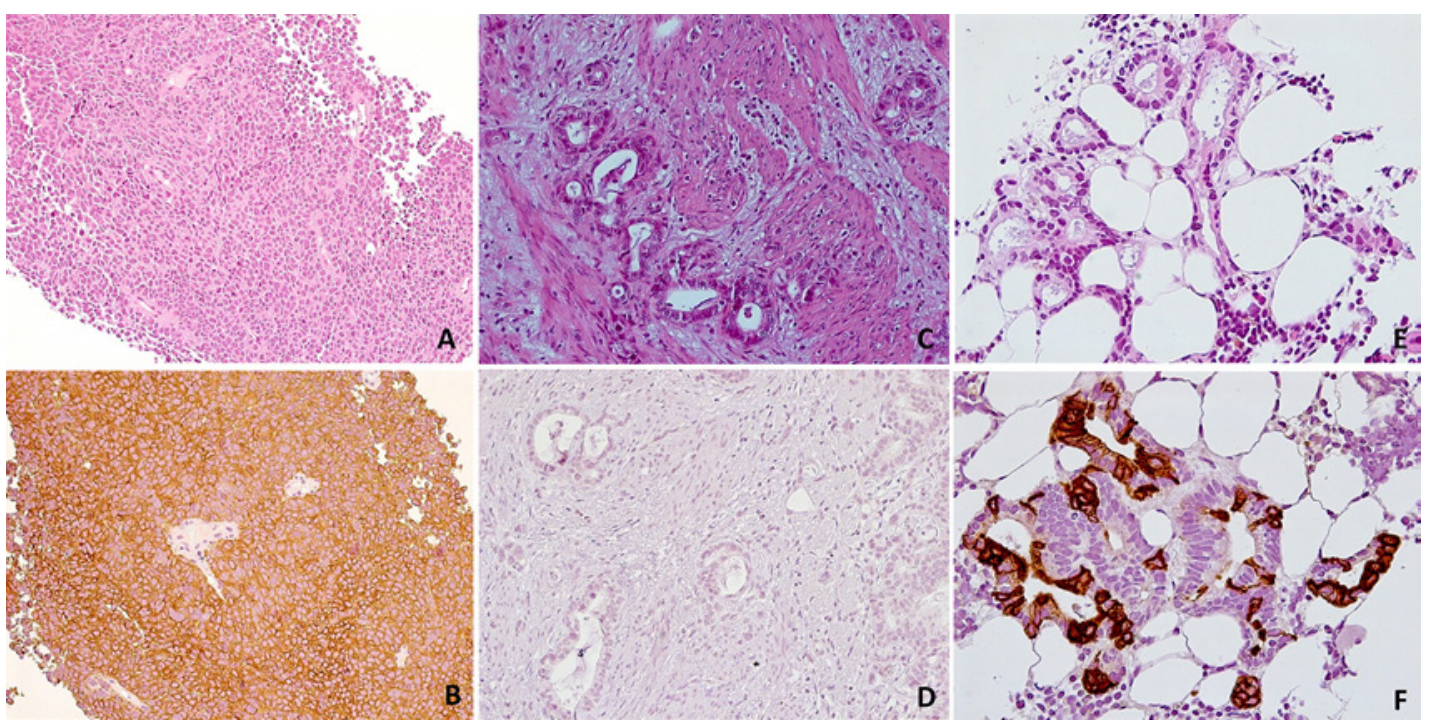

Fig. 2. Small-cell carcinoma in a biopsy from the pancreatic head (HE stain. Original magnification, $\times 200$; A) with diffuse expression of synaptophysin (immunohistochemistry. Original magnification, $\times 200$; B). Infiltrating adenocarcinoma of the gallbladder showing lack of expression of neuroendocrine markers (HE stain. Original magnification, $\times 200$; C) including negativity for synaptophysin (immunohistochemistry. Original magnification, $\times 200$; D). Metastasis of the adenocarcinoma of the gallbladder in the os ilium (HE stain. Original magnification, $\times 400$; E) with patchy CK20 expression (immunohistochemistry. Original magnification, $\times 400 ; \mathbf{F})$.

at a reduced dose (carboplatin AUC 4 and etoposide $80 \mathrm{mg} / \mathrm{m}^{2}$ ) due to a low initial PS and poor tolerance with profound fatigue during chemotherapy. The symptoms rapidly improved after initiation of chemotherapy and the laboratory parameters normalized after the second chemotherapy cycle.

Follow-up CT scan revealed a partial response after the first 3 cycles of chemotherapy with complete regression of the tumor after completion of chemotherapy. Subsequent CTs confirmed complete tumor regression. An intense follow-up with CTs every 3 months for the first 2 years was planned.

The first follow-up CT scan performed 3 months after the completion of chemotherapy demonstrated a thickening of the gallbladder wall, consistent with cholecystolithiasis which had already been documented in previous images. Another CT scan 3 months later showed a 5 -mm concrement in the gallbladder which increased to $9 \mathrm{~mm}$ on a consecutive CT performed after further 3 months. An ultrasound of the gallbladder revealed a polypoid structure with a maximum diameter of $17 \mathrm{~mm}$. We discussed the possibility of a second malignancy and the option of surgery, but the patient refused a cholecystectomy at that point and agreed to a follow-up CT scan. A CT scan performed 4 months later showed a $2.3 \times 2.3-\mathrm{cm}$ tumor with no other abnormalities (Fig. 3). At that point we could not rule out a second malignancy and the patient consented to a cholecystectomy. A PET/CT was performed prior to the operation in order to exclude metastatic disease.

Histopathological examination of the gallbladder specimen revealed a poorly differentiated adenocarcinoma (Fig. 2C) arising from high-grade biliary intraepithelial neoplasia of the mucosal epithelium. No SCLC components could be identified and the tumor was consistently negative for the neuroendocrine marker synaptophysin (Fig. 2D) with co-expression of CK7 and CK20. The gallbladder cancer was resected at an early stage (pT2a) without any 
Fig. 3. Gallbladder adenocarcinoma with increased ${ }^{18} \mathrm{~F}$-fluorodeoxyglucose metabolic activity (standardized uptake value maximum 19.18) in PET/ CT.

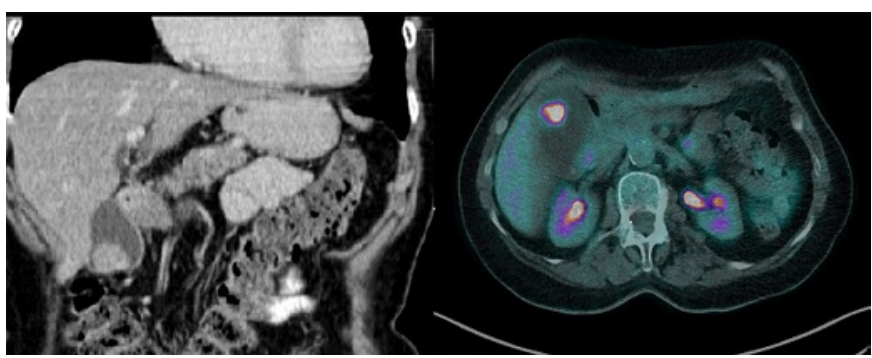

lymph node metastases or presence of any risk factors, so an adjuvant treatment was not recommended. The patient quickly recovered from cholecystectomy and was seen every 3 months for follow-up consultation having an ECOG PS of 0 . Three years after the diagnosis of the PSCC and 20 months after the resection of the gallbladder cancer, the patient presented with fatigue, generalized skeletal pain, and rapid clinical deterioration. PET/CT revealed diffuse bone and liver metastases. Histopathological examination of a bone marrow biopsy unfortunately showed infiltration by an adenocarcinoma, histomorphologically and immunohistochemically consistent with relapse of the gallbladder cancer (Fig. 2E, F). A palliative chemotherapy with gemcitabine is currently planned.

\section{Discussion}

The exact incidence of PSCC is unknown. Ivanics et al. [1] reported that PSCC accounted for $0.2 \%$ of all pancreatic tumors. According to the literature, PSCC is characterized by an aggressive disease course and extremely poor prognosis [2]. It is histopathologically indistinguishable from SCLC but may be assigned to the organ of origin by immunohistochemical examinations. Due to lack of data on the tumor behavior, treatment is extrapolated from the management of SCLC. As for other extrapulmonary small-cell carcinomas, there have been different approaches suggested for patients with localized disease with frequent use of multimodal treatment. For those with extensive disease, chemotherapy in analogy to treatment on SCLC has been applied in the vast majority of cases.

The current case resembles most of the cases described so far with a rapid onset of symptoms consistent with the aggressive clinical course of this disease and advanced stage at diagnosis. Ichikawa et al. [3] reported characteristic CT findings in 3 patients with PSCC showing a large tumor mass with homogeneous, slight contrast enhancement and confluent lymphadenopathy of regional lymph nodes. This description is consistent with other reports [4], as well as with the characteristics found in this case. Frequent localization in the pancreatic head has been reported but generally attempts to characterize the disease are limited due to the small number of patients.

Reviewing the literature, response to treatment is usually poor with survival of a few months reported in most of the cases. Isolated reports of a patient who achieved complete remission with cisplatin and etoposide [5] as well as another report of 2 patients achieving complete remission after treatment with carboplatin and etoposide [4] demonstrate the poor outcome for this disease. Although there is no standard treatment in the management of PSCC, and application of various chemotherapy regimens such as gemcitabine or the combination of cisplatin and irinotecan have been attempted [6,7], the combination of cisplatin and etoposide is the most frequently used treatment [2]. In recent years, response to immunotherapy has been reported with a partial response to late line treatment with nivolumab [8] as well as a complete response with the combination of ipilimumab and nivolumab [9]. We 
chose to treat this patient with carboplatin due to the better toxicity profile compared to cisplatin. Our patient remains in complete remission with regards to PSCC 3 years after the completion of chemotherapy. She tolerated treatment well and had an ECOG PS of 0, with an excellent quality of life so far. This case is remarkable regarding the fact that the patient was diagnosed with a second aggressive malignancy with a completely distinct histology that unfortunately relapsed within 20 months with widespread hematogenous metastasis.

\section{Conclusion}

This case supports treatment of PSCC with a platin-based combination with etoposide in concordance with previous case reports.

\section{Statement of Ethics}

Written informed consent was obtained from the patient for publication of this case report including accompanying images. A copy of the written consent is available for review by the Editor-in-Chief of this journal.

\section{Conflict of Interest Statement}

The authors have no conflicts of interest to declare.

\section{Funding Sources}

There are no funding sources to declare.

\section{Author Contributions}

K.T.: medical oncologist in charge of the patient. S.S.: pathologist in charge of the tumor specimen analyses. K.T. wrote the manuscript. S.S. and S.P. critically revised the manuscript. All authors read and approved the final manuscript.

\section{References}

1 Ivanics T, Bergquist JR, Shubert CR, Smoot RL, Habermann EB, Truty MJ. Small cell carcinoma of the pancreas: a surgical disease. Pancreas. 2016 Nov;45(10):1461-6.

2 Vos B, Awada A, Hendlisz A. Primary small-cell carcinoma of the pancreas: an extensive review of the literature. Pancreatology. 2004;4:521-6.

3 Ichikawa T, Federle MP, Ohba S, Ohtomo K, Sugiyama A, Fujimoto H, et al. Atypical exocrine and endocrine pancreatic tumors (anaplastic, small cell, and giant cell types): CT and pathologic features in 14 patients. Abdom Imaging. Jul-Aug 2000;25(4):409-19.

4 Sakamoto H, Kitano M, Komaki T, Noda K, Chikugo T, Kudo M. Small cell carcinoma of the pancreas: role of EUS-FNA and subsequent effective chemotherapy using carboplatin and etoposide. J Gastroenterol. 2009; 44(5):432-8.

5 Morant R, Bruckner HW. Complete remission of refractory small cell carcinoma of the pancreas with cisplatin and etoposide. Cancer. 1989 Nov 15;64(10):2007-9.

6 Sannomiya H, Okamoto T, Ryu S, Ichikawa J, Koreeda N, Shin Y, et al. [A case of small cell carcinoma of the pancreas]. Gan to Kagaku Ryoho. 2019 Dec;46(13):2312-4. 
7 Berkel S, Hummel F, Gaa J, Back W, Hofheinz R, Queisser W, et al. Poorly differentiated small cell carcinoma of the pancreas. A case report and review of the literature. Pancreatology. 2004;4(6):521-6.

8 Ugwu JK, Nwanyanwu C, Shelke AR. Dramatic response of a metastatic primary small-cell carcinoma of the pancreas to a trial of immunotherapy with nivolumab: a case report. Case Rep Oncol. 2017;10(2): 720-5.

9 Husnain M, Park W, Ramos JC, Johnson TE, Chan J, Dasari A, et al. Complete response to ipilimumab and nivolumab therapy in a patient with extensive extrapulmonary high-grade small cell carcinoma of the pancreas and HIV infection. J Immunother Cancer. 2018 Jul 9;6(1):66. 